\title{
The internist in the surgical setting: results from the Italian FADOI-ER survey
}

\author{
Fabio Gilioli, ${ }^{1}$ Carlo Di Donato, ${ }^{1}$ Vincenzo Ferrari, ${ }^{1}$ Marcello Bertesi,${ }^{1}$ Giuseppe Chesi ${ }^{2}$ \\ ${ }^{1}$ UO di Medicina Interna, Ospedale "B. Ramazzini”, Carpi (MO); ${ }^{2}$ UO di Medicina Interna, Ospedale “C. Magati”, Scandiano \\ (RE), Italy
}

\begin{abstract}
More and more frequently, patients admitted to surgical wards present characteristics similar to those admitted to medical units. They are fragile patients, often elderly, with significant comorbidity. In recent years, to address these emerging clinical issues in a surgical setting, different organizational models involving specialists of different backgrounds were studied, and in particular involving internists and geriatricians. To widen our current knowledge, in 2011 the Federation of Associations of Hospital Doctors on Internal Medicine of Emilia-Romagna, northern Italy (FADOI-ER), proposed a questionnaire to the public healthcare internal medicine departments of the Emilia Romagna region to collect information as to in what way and to what extent internists are involved in the management of surgical patients. In this article, we analyze the results of the questionnaire and make some organizational considerations and proposals. The questionnaire was very simple, consisting of 14 items. The survey was conducted from 1-28 February 2011. Replies were received from 20 internal medicine departments of a total of 75 in the Emilia Romagna region. The FADOI-ER survey has some limitations, the first of which is that only just under $25 \%$ of internal medicine departments in the Emilia Romagna region took part. However, the results are still interesting and seem to suggest that internists, because of their particular cultural background and training, could be the preferred partners for comanagement within the context of inpatient surgical procedures. The results of the FADOI-ER questionnaire are also consistent with the data reported in literature and daily clinical experience that highlight the need for a more multi-specialist approach to patient management with medical internists. Further studies will help provide answers as to the best way to conduct this multidisciplinary approach that could represent one of the future challenges for healthcare.
\end{abstract}

\section{Introduction}

More and more frequently, patients admitted to surgical wards present characteristics similar to those admitted to medical units. ${ }^{1}$ As Mazzi reminded us, these are fragile patients, ${ }^{2}$ often elderly with significant morbidity. This is confirmed by results of various studies. Jencks ${ }^{3}$ reported that, in 2003 in the USA,

Correspondence: Fabio Gilioli, UO di Medicina Interna, Ospedale "B. Ramazzini”, via Molinari 2, 41012 Carpi (MO), Italy.

Tel. +39.59659309 - +39.3496995740.

E-mail: f.gilioli@ausl.mo.it

Key words: internal medicine, hospital management, orthogeriatric care model, hospitalist model.

Conflict of interests: the authors declare no potential conflict of interests.

This work is licensed under a Creative Commons Attribution NonCommercial 3.0 License (CC BY-NC 3.0).

CCopyright F. Gilioli et al., 2013

Licensee PAGEPress, Italy

Italian Journal of Medicine 2013; 7:32-38

doi:10.4081/itjm.2013.32 among patients treated under the Medicare program who were sent home from surgery units and then readmitted within 30 days, $70.5 \%$ of cases were mainly characterized by medical pathologies such as heart failure, pneumonia, gastrointestinal pathologies and sepsis. Today, in an orthopedic setting, mortality for fracture of the femur is still $10 \%$ at one month after surgery and is correlated with pre-operative comorbidity, and functional, cognitive and nutritional patient status. ${ }^{4-6}$ The main causes of death are heart failure and infective pulmonary pathologies. ${ }^{7,8}$ It has also been observed that only $10 \%$ of orthopedic patients admitted are completely autonomous and without any comorbidity, while another $10 \%$ is made up of patients with motor disability ranging from serious to bed-ridden. The remaining $80 \%$ were autonomous before the fracture but had functional limitations that compromised daily routine activities. ${ }^{9-11}$ In recent years, in order to deal with these emerging clinical issues in a surgical setting, various organizational models have been studied that involve specialists from different fields, but in particular internists and geriatricians. In order to explore the problems involved, the Federation of Associations of Hospital Doctors on Internal Medicine of Emilia-Romagna, northern Italy (FADOI-ER) proposed a questionnaire to the public healthcare internal medicine departments in the Emilia Romagna region. 
The survey investigated in what way and to what extent internists are involved in the management of surgical patients.

\section{Materials and Methods}

In 2011, the FADOI-ER proposed a very simple questionnaire consisting of 14 items (Table 1). The survey was carried out 1-28 February 2011 and aimed to collect information on the consultancy role of internists in a surgical setting. The questions were formulated to evaluate: i) the integrated healthcare models adopted; ii) the use of human resources in relation to the type of healthcare model proposed; iii) any possible involvement of other internists in the integrated healthcare model.

\section{Results}

Response to the survey was received from 20 internal medicine departments of a total of 75 in Emilia Romagna (Table 2). Of these, $75 \%$ had $30-60$ beds (Table 1) and $70 \%$ of the departments involved had less than 300 beds (Table 1). In all hospitals whose internal medicine departments took part in the survey, the main surgical wards were: general surgery (100\%), orthopedics (85\%), gynecology (65\%), urology $(60 \%)$, otolaryngology $(60 \%)$, ophthalmology $(60 \%)$. From the answers to Question 6, How often are internal medicine specialists consulted about surgery? (Figure 1), it can be seen that in $60 \%$ of cases an internist is available in the surgical setting on a weekly basis and 2-3 times per week in 35\% of cases. Also in relation to how internists are distributed around the hospital, the main requests for consultancy were made by general surgery (45\%), orthopedics (40\%) and urology (10\%) departments (Figure 2). Fifty percent of internal medicine departments that responded to the survey have created or are in the process of creating programmed Internal Medicine services within their surgical unit (Table 1). Among the internal medicine departments that incorporate daily structured consultancy (Figure 3), in most cases $(60 \%)$, it is estimated that medical personnel are involved for 1-2 $\mathrm{h}$ a day. In cases in which consultancy services are not included in any codified program, medical personnel are involved for less than $60 \mathrm{~min}$ a day $(60 \%)$ (Figure 4). Other medical specialities that were reported to provide consultancy services in the surgical unit were cardiology $(100 \%)$, nephrology $(50 \%)$ and pneumology $(20 \%)$. The geriatric department, available in $45 \%$ of the hospitals taking part in the questionnaire (Table 1), seems to be less involved in consultancy services within surgical units $(10 \%)$. Interestingly, in $25 \%$ of cases, and particu- larly in the smaller hospitals, the internist was described as the only consultant available.

\section{Discussion}

\section{Data and trends}

The FADOI-ER survey has some limitations, the first being that only just under $25 \%$ of internal medicine departments in the Emilia Romagna region took part. Furthermore, it is probable that the hospital services that answered the questionnaire were those who

Table 1. Results of the FADOI questionnaire carried out in Emilia Romagna, northern Italy, on internal medicine interventions in a surgical setting.

1) How many beds does your hospital have?
a) $<200$
b) $200-300$
$40 \%$
c) $>300$
$30 \%$
$30 \%$

2) How many beds does the Internal Medicine Department have? a) $<30$ b) $30-60$ $5 \%$ c) $>60$ $75 \%$

3) Does your hospital have a geriatric department?

$\begin{array}{ll}\text { a) Yes } & 45 \% \\ \text { b) No } & 55 \%\end{array}$

4) Which surgical specialities are available in your hospital? a) General surgery $\quad 100 \%$ b) Orthopedics $\quad 85 \%$ c) Urology $\quad 60 \%$ d) Obstetrics-Gynecology $\quad 65 \%$ e) Otolaryngology $\quad 60 \%$ f) Ophthalmology $\quad 60 \%$ g) Other $0 \%$

5) Are internal medicine specialists consulted in a surgical setting? a) Yes $\quad 100 \%$

b) No $\quad 0 \%$

6) If yes, how often?

a) 2-3 times a week $\quad 35 \%$

b) At least once a day $\quad 60 \%$

c) More than once a day $5 \%$

7) Which departments request internal medicine consultancy the most (list at least 3 departments and give an estimate of number of requests, \%)?
a) $1^{\text {st }}$ Surgery
$45 \%$
b) $2^{\text {nd }}$ Orthopedics
$40 \%$
c) $3^{\text {rd }}$ Urology
$10 \%$

8) Have any surgical units incorporated programmed internal medicine services within their unit?
a) Yes
$50 \%$
b) No
$50 \%$

9) If yes, which models were used?

a) Programmed daily consultancy

b) Pre- and postoperative services managed within the surgical unit

c) Creation of multi-specialist teams

$30 \%$ $30 \%$ 
Table 1. Continued from previous page.

10) If yes, which surgical units were involved?

$\begin{array}{lr}\text { a) General surgery } & 40 \% \\ \text { b) Orthopedics } & 100 \% \\ \text { c) Urology } & 10 \% \\ \text { d) Obstetrics-Gynecology } & 10 \% \\ \text { e) Other } & 10 \%\end{array}$

11) If the programmed consultancies listed above are operative, how long are internists involved for?
a) A doctor $1-2 \mathrm{~h}$
$60 \%$
b) A doctor $<60 \mathrm{~min}$
$30 \%$
c) A doctor 2-4 h
$10 \%$
d) A doctor $>4 \mathrm{~h} / \mathrm{die}$
$0 \%$

12) If your unit does NOT have a consultancy program as listed above how much time is staff expected to dedicate to such a service?
a) 1 doctor $<60 \mathrm{~min}$
$60 \%$
b) 1 doctor $<30 \mathrm{~min}$
$40 \%$
c) 1 doctor $>60 \mathrm{~min}$
$0 \%$

13) In your hospital, what other departments contribute to the management of medical complications presented in the surgical unit?
a) Cardiology
$100 \%$
b) Nephrology
$50 \%$
c) Geriatrics
$10 \%$
d) Pneumology
$20 \%$
e) Other
$20 \%$
f) None
$25 \%$

14) What contribution do they make?

a) Consultant on call

$95 \%$

b) Organizational models with programmed services (specify department) already had internists involved in the surgical setting and this could be considered a quite significant selection bias. In spite of the limited representation of the samples studied by the questionnaire, our results are similar to those reported in the literature. Since 2001, in the USA, requests for an approach involving surgeons and medical specialists have been constantly on the increase with $35-40 \%$ of hospitalized patients managed in this way. ${ }^{12}$ One of the most studied management models is that represented by the co-management between the surgeon and the medicine physician, interpreted to mean an internist, geriatrician or internal medicine specialist. Such a specialist would describe his role as that of the daily management of chronic medical comorbidities and possible acute complications of the surgical patient. ${ }^{12,13}$ Some years ago in the USA, the role of hospitalist was created. These were physicians mainly specialized in internal medicine who, according to the original definition, should carry out at least $25 \%$ of their work in assisting hospitalized patients on internal medicine issues. ${ }^{14}$ This role has progressively widened its scope to include surgical patients. This has had an extremely favorable impact on clinical practice, reducing the average length of hospital stay without any increase in the number of readmissions or mortality rates. ${ }^{12,15-17}$ Furthermore, in the orthopedic setting, more complex care models have

Table 2. List of public healthcare departments of Internal Medicine that completed the FADOI-ER questionnaire.

\begin{tabular}{|c|c|}
\hline 1) & UU.OO. Medicina Interna Ospedale Budrio (Bologna) \\
\hline 2) & UU.OO. Medicina Interna Ospedale, Riccione (Rimini) \\
\hline 3) & UU.OO. Medicina Interna Ospedale, Bagno di Romagna (Forlì Cesena) \\
\hline 4) & UU.OO. Medicina Interna Ospedale, Imola (Bologna) \\
\hline 5) & UU.OO. Medicina Interna Ospedale, Castel San Giovanni (Piacenza) \\
\hline 6) & UU.OO. Medicina ad Alta Rotazione Ospedale, Ferrara \\
\hline 7) & UU.OO. Medicina Interna III Ospedale, Reggio Emilia \\
\hline 8) & UU.OO. Medicina Interna Ospedale, Faenza (Ravenna) \\
\hline 9) & UU.OO. Medicina Interna Ospedale San Giovanni in Persiceto (Bologna) \\
\hline 10) & UU.OO. Medicina Interna Ospedale Sant'Arcangelo, Romagna (Rimini) \\
\hline 11) & Clinica Medica II Ospedale, Ferrara \\
\hline 12) & UU.OO. Medicina Critica, Piacenza \\
\hline 13) & UU.OO. Medicina Interna Ospedale, Carpi (Modena) \\
\hline 14) & UU.OO. Medicina Interna Ospedale, Pavullo (Modena) \\
\hline 15) & UU.OO. Medicina Interna Ospedale, Vignola (Modena) \\
\hline 16) & UU.OO. Medicina Interna Ospedale, Sassuolo (Modena) \\
\hline 17) & UU.OO. Medicina Interna Ospedale, Porretta (Bologna) \\
\hline 18) & UU.OO. Medicina d'Urgenza Ospedale civile, Modena \\
\hline 19) & UU.OO. Medicina Interna I Ospedale, Reggio Emilia \\
\hline 20) & UU.OO. Medicina Interna II Ospedale, Fidenza \\
\hline
\end{tabular}


been studied in the light of this type of experience. These models are based on setting up a group made up of different professional figures (orthopedic surgeons, geriatricians, nurses, physiatrists) capable of creating a true orthogeriatric structure. ${ }^{18,19}$ The results of our questionnaire obviously provide a best case scenario of the most advanced strategies and most motivated staff in this context. Similar to our findings, the co-management of patients undergoing surgery is mostly centered on the hospitalist, internist and geriatrician, a model that has become more familiar over the last 15 years. ${ }^{12,20}$ The move towards this kind of approach is also probably due to the opportunity it offers to simplify organizational issues.

\section{Experiences and care settings proposed in the literature}

An analysis of all the studies carried out so far shows there have been few randomized trials on this

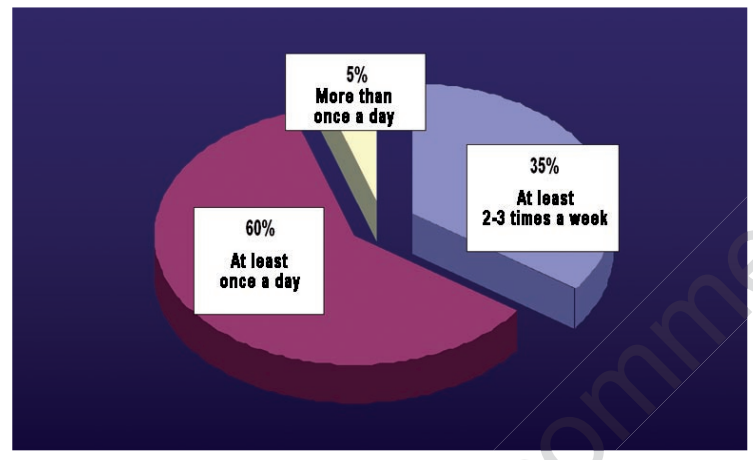

Figure 1. Answers (\%) to Question 6.

How often are internal medicine specialists consulted in a surgical setting?

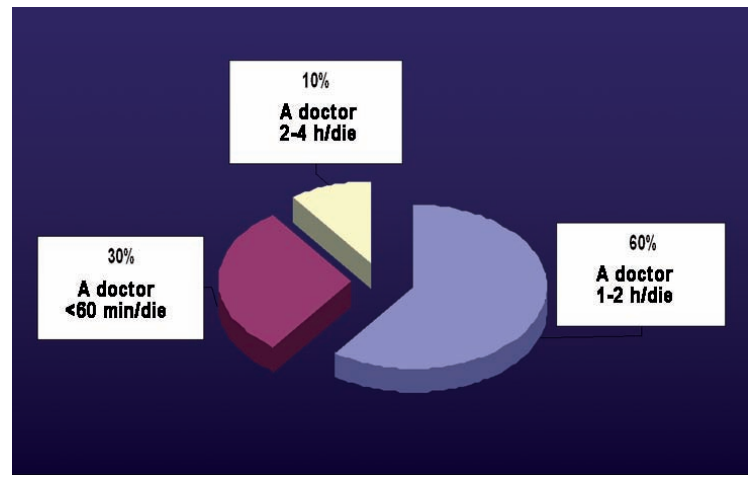

Figure 3. Answers (\%) to Question 11.

If the programmed consultancies (listed in Question 9) are operative, how long are internists involved for? issue and that studies were for the most part conducted in an orthopedic setting. This makes it difficult to draw definitive conclusions concerning the efficacy of the different management models ${ }^{21}$ (see also the interesting article recently published by Colombo in this Journal). ${ }^{22}$ It is, in any case, useful to highlight the results obtained in the most important clinical studies.

\section{Orthopedic surgery}

In 2001, Marcantonio et al., ${ }^{23}$ in a randomized study of patients with hip fracture, showed that, compared with traditional care, co-management of the geriatric patient significantly reduced the number and the seriousness of episodes of delirium. In a more recent prospective observational study in Australia, ${ }^{24}$ Fisher et al. compared 447 patients with hip fracture cared for under an orthogeriatric co-management program to 504 patients followed for three years be-

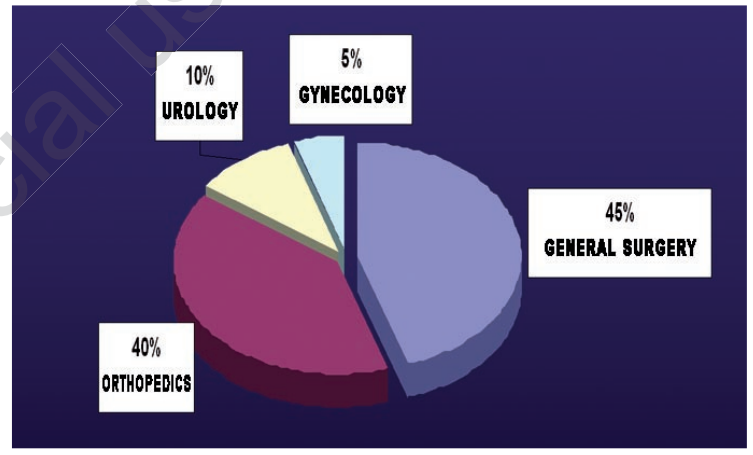

Figure 2. Answers (\%) to Question 7.

Which departments request internal medicine consultancy the most?

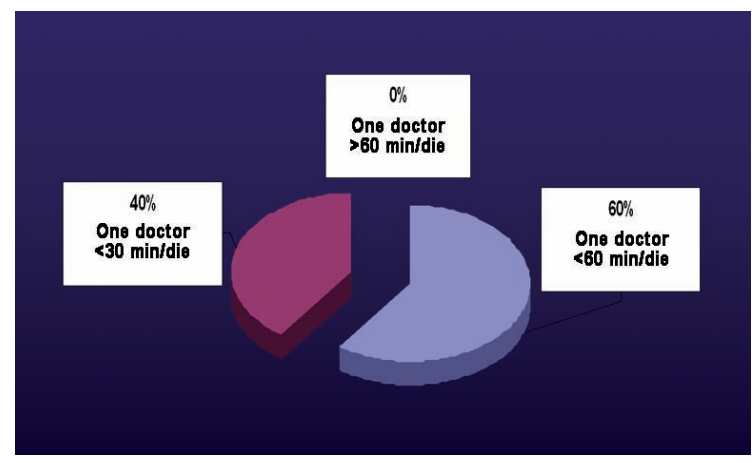

Figure 4. Answers (\%) to Question 12.

If your unit does NOT have a consultancy program (listed in Question 9) how much time is staff expected to dedicate to such a service? 
fore the program was set up. Post-operative medical complications and re-admission rates at six months were significantly reduced in the orthogeriatric comanagement group. In a retrospective study published in 2009, Friedman et al. ${ }^{25}$ compared 163 patients over 60 years of age with fracture of the femur cared for under an orthogeriatric co-management program with 121 patients under standard care. The co-management care group developed fewer post-operative infections and complex complications (delirium, heart problems, thromboembolism) along with a shorter hospital stay. There were no differences in mortality either in hospital or at 30 days or in hospital readmission rates.

In 2004, Huddleston et al. ${ }^{26}$ carried out a randomized controlled trial on 526 patients undergoing elective surgery for complete hip or knee replacement. They compared co-management by a hospitalist and an orthopedic surgeon with standard care based on consultancy intervention on request. Patients followed by a hospitalist had a higher probability of leaving hospital without post-operative complications. There was no difference in mortality rates or in total cost of treatment between the two care models. In a second study, in 2005, Phy et al. ${ }^{27}$ analyzed 466 patients over 65 years of age admitted for hip fracture. The patients in the co-management group underwent surgery quicker and had a shorter average hospital stay. There were no differences in hospital mortality or in readmission at 30 days.

\section{Cardiac surgery and neurosurgery}

In 1990, Macpherson et al. ${ }^{28}$ evaluated internist comanagement of 165 patients undergoing cardiothoracic surgery. The authors showed that, compared with the previous year, the setting up of the internist comanagement program was associated with a reduction of six days in the length of hospital stay, fewer laboratory tests and radiological examinations, and a trend towards lower mortality. In 2010, Auerbach et al. ${ }^{29}$ carried out an observational study on the effects of comanagement with a hospitalist of neurosurgical patients examining the level of professional satisfaction, length of hospital stay, readmission rates, mortality rates and cost. They concluded that health professionals expressed greater satisfaction in the care provided and that costs were reduced (approx. $\$ 1500$ per patient) but there was no improvement in results of other outcomes.

\section{General considerations and future prospects}

It seems clear that, in most cases, all these studies concern and involve co-management and an important contribution from physicians who, whatever the terminology used, can be best described as in- ternists. In fact, it is problematic for the specialist, who is usually only involved in well-defined clinical situations, to take responsibility for an overall evaluation of surgical patients who, as we said earlier, present complex and multiple comorbidities. Epidemiological data reported by Gulsham et al. ${ }^{12}$ seem to support these observations. In this century in the USA, the generalist physicians, mostly made up of internists, geriatricians or general medicine doctors, are of increasing importance in hospital comanagement in the surgical setting while specialists have a progressively smaller role. Scientific evidence and the results of the FADOI-ER survey seem to suggest that internists, in virtue of their particular cultural background, their wider general training, and their presence in even the smallest hospitals have a privileged role to play in co-management programs on surgical wards. Such a role can be designed and adapted according to the characteristics and requirements of each hospital. It would, therefore, be useful to validate this new organizational approach also in Italy and in Europe as a whole in controlled clinical trials.

Other data to emerge, in particular in Italy, show that structural and organizational changes of this type need to look at hospital staffing to ensure that more internists are available on the wards. ${ }^{12,30}$ Another factor to emerge from the literature is how any changes in a surgical setting need the full involvement of all healthcare professionals involved and a change in their cultural attitudes. ${ }^{31-33}$ Given this, from our questionnaire it emerges that $60 \%$ of the internal medicine departments that responded to the survey confirmed that internists were involved in consultancy services for 1-2 $h$ per day. It could, therefore, be hypothesized that in the future more time could be spent in providing these services. Such a commitment cannot be sustained unless resources are redistributed according to new organizational strategies that are not based on specialized expertise but focused on the patient and his or her needs. This could also eventually be applied to the organization structured to provide greater intensity of care. Another less costly method from an organizational point of view could be to identify surgical patients who require daily clinical evaluation using a score system based, for example, on risk factors that have already been partially recognized., ${ }^{4,34}$ This could limit the biggest part of the clinical workload to some patient subgroups.

\section{Models for the future}

We can identify organizational models that could be applied in hospitals in the future. Although these models, however flexible they may be, will obviously be related to the different characteristics and requirements of each hospital, they should lead to a 
constant improvement in the synergy between professionals and an increased presence of internists in surgical units. A first model, called structured consultancy, could be applied to any hospital. This is very similar to some of the models that have also emerged from our survey. Structured consultancy involves establishing a timetable in which the internist is available for partial or complete medical examinations either alone or together with a specialized surgeon. In this context, the internist, either independently or together with the surgeon, will be responsible for the management of issues that may not necessarily be related to the surgical intervention itself or its local consequences. On the other hand, a second model that could also be applied in hospitals of any size could be to put the management of patients admitted to surgical or polyspecialist units completely in the hands of an internal medicine department. This would leave the surgeon to deal only with consultancy services for the surgical intervention itself and wound management. Obviously, this type of model requires a huge step forward in the development of clinical governance. It would be a highly suitable approach in the context of hospital organization aimed at improving intensity of care. This is currently considered a particularly efficient and valid approach to overall care of elderly, complex patients with polypathologies. ${ }^{35}$

\section{Conclusions}

Over recent years, the number of fragile patients has increased and this is changing the scene of the clinical and general care of these patients in surgical settings. The complexity of this epidemiological change will have a significant impact and, even though these changes are still as yet undefined, they can be expected to also affect surgical outcome. Results from the FADOI-ER questionnaire agree with data from the literature and from daily clinical experience. They underline the need for greater collaboration between specialist surgeons and internists in patient care.

We have proposed two models that are in line with this type of organization. One represents structured consultancy, a model that could be applied in any hospital, and one that could be integrated into hospital reorganization strategies that aim to increase intensity of care. This second model foresees management of patients in a surgical unit by internists. In this case, the specialist surgeon would provide only consultancy services relating to the surgical intervention itself and its local consequences.

Further studies will be needed to identify which of these multidisciplinary healthcare models could best present the challenge for the near future.

\section{References}

1. Nardi R, Scanelli G, Borioni D, et al. The assessment of complexity in internal medicine patients. The FADOI Medicomplex Study. Eur J Intern Med 2007;18:283-7.

2. Mazzi G. Simultaneous care and frail patients: are we on target? Ital J Med 2011:5:79-81.

3. Jencks SF, Williams MV, Coleman EA. Rehospitalizations among patients in the Medicare Fee-for-Service Program. N Engl J Med 2009;360:1418-28.

4. Farahmand BY, Michaelsson K, Ahlbom A, et al. Swedish Hip Fracture Study Group. Survival after hip fracture. Osteoporos Int 2005;16:1583-90.

5. Empana JP, Dargent-Molina P, Breart G, EPIDOS Group. Effect of hip fracture on mortality in elderly women: the EPIDOS prospective study. J Am Geriatr Soc 2004;52:685-90.

6. Holvik K, Ranhoff AH, Martinsen MI, Solheim LF. Predictors of mortality in older hip fracture inpatients admitted to an ortho-geriatric unit in Oslo, Norway. J Aging Health 2010;22:1114-31.

7. Cameron ID, Chen JS, March LM, et al. Hip fracture causes excess mortality owing to cardiovascular and infectious disease in institutionalized older people: a prospective 5-year study. J Bone Miner Res 2010; 25:866-72.

8. Roche JJW, Wenn RT, Sahota O, Moran CG. Effect of comorbidities and postoperative complications on mortality after hip fracture in elderly people: prospective observational cohort study. BMJ 2005;331:1374-6.

9. Kristensen MT, Foss NB, Ekdahl C, Kehlet H. Prefracture functional level evaluated by the New Mobility Score predicts in-hospital outcome after hip fracture surgery. Acta Orthop 2010;81:296-302.

10. Penrod JD, Litke A, Hawkes WG, et al. Heterogeneity in hip fracture patients: age, functional status, and comorbidity. J Am Geriatr Soc 2007;55:407-13.

11. Ranhoff AH, Holvik K, Martinsen MI, et al. Older hip fracture patients:three groups with different needs. BMC Geriatr 2010;10:65.

12. Sharma G, Kuo Y-F, Freeman J, et al. Comanagement of hospitalized surgical patients by medicine physicians in the United States. Arch Intern Med 2010;170:363-8.

13. Whinney C, Michota F. Surgical comanagement: a natural evolution of hospitalist practice. J Hosp Med 2008;3:394-7.

14. Wachter RM. The evolution of the hospitalist model in the United States. Med Clin North Am 2002;86:687-706.

15. Peterrson MC. A systematic review of outcomes and quality measures in adult patients cared for by hospitalists vs nonhospitalists. Mayo Clin Proc 2009;84:248-54.

16. Lindenauer PK, Rothberg MB, Pekow PS, et al. Outcomes of care bay hospitalist, general internist, and family physicians. N Engl J Med 2007;357:2589-600.

17. Roy A, Heckman MG, Roy V. Associations between the hospitalist model of care and Quality-of-Care-Related outcomes in patients undergoing hip fracture surgery. Mayo Clin Proc 2006;81:28-31.

18. Pioli G, Giusti A, Barone A. Orthogeriatric care for the elderly with hip fractures: where are we? Aging Clin Exp Res 2008;20:113-22.

19. Frondini C, Lunardelli ML. Ortogeriatria: un nuovo mo- 
dello di assistenza ai pazienti anziani con frattura di femore e comorbilità. Ital J Med 2010;4:105-10.

20. Kuo Y-F, Sharma G, Freeman JL, Goodwin JS. Growth in the care of older patients by hospitalits in the United States. N Eng J Med 2009;360:1102-12.

21. Chong CP, Savige J, Lim WK. Orthopaedic-geriatric models of care and their effectiveness. Australas J Ageing 2009:171-6.

22. Colombo F. Il medico internista e l'Ortopedia: quali competenze, quali modelli? Ital J Med 2010;4:81-3.

23. Marcantonio ER, Flacker JM, Wright RJ, Resnick NM. Reducing delirium after hip fracture: a randomized trial. J Am Geriatr Soc 2001;49:516-22.

24. Fisher AA, Davis MW, Rubenach SE, et al. Outcomes for older patients with hip fractures: the impact of orthopedic and geriatric medicine cocare. J Orthopaed Trauma 2006;20:172-8.

25. Friedman SM, Mendelson DA, Bingham KW, Kates SL. Impact of a comanaged geriatric fracture center on shortterm hip fracture outcomes. Arch Intern Med 2009;169:1712-7.

26. Huddleston JM, Long KH, Naessens JM, et al. Medical and surgical comanagement after elective hip and knee arthroplasty: a randomized, controlled trial. Ann Intern Med 2004;141:28-38.

27. Phy MP, Vanness DJ, Melton LJ 3rd, et al. Effects of a hospitalist model on elderly patients with hip fracture. Arch Intern Med 2005;165:796-801.
28. Macpherson DS, Parenti C, Nee J, et al. An internist joins the surgery service: does comanagement make a difference? J Gen Intern Med 1994;9:440-4.

29. Auerbach AD, Wachter RM, Cheng HQ, et al. Comanagement of surgical patients between neurosurgeons and hospitalists. Arch Intern Med 2010;170:2004-10.

30. O'Malley PG. Internal medicine comanagement of surgical patients: can we afford to do this? Arch Intern Med 2010;170:1965-6.

31. Kates SL, Mendelson DA, Friedman SM. Co-managed care for fragility hip fractures (Rochester model). Osteoporos Int 2010;21:S621-5.

32. Auerbach AD, Rasic MA, Sehgal N, et al. Opportunity missed: medical consultation, resource use, and quality of care of patients undergoing major surgery. Arch Intern Med 2007;167:2338-44.

33. Naglie G, Tansey C, Kirkland JL, et al. Interdisciplinary inpatient care for elderly people with hip fracture: a randomized controlled trial. Can Med Assoc J 2002; 167:25-32.

34. Ranhoff AH, Holvik K, Martinsen MI, et al. Older hip fracture patients: three groups with different needs. BMC Geriatrics 2010;10:65.

35. Cioni G, Josa G, Chesi G, et al. Medicina Interna ed organizzazione ospedaliera per intensità di cure: la posizione di FADOI Emilia Romagna. Ital J Med 2012; 6:72-4. 